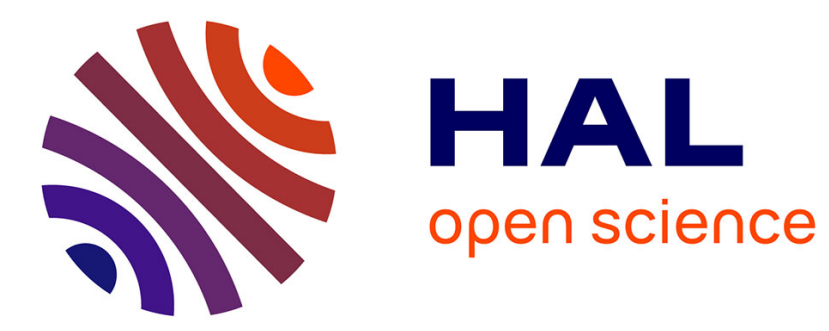

\title{
Efficient routing protocol for concave unstable terahertz nanonetworks
}

\author{
Lina Aliouat, Hakim Mabed, Julien Bourgeois
}

\section{To cite this version:}

Lina Aliouat, Hakim Mabed, Julien Bourgeois. Efficient routing protocol for concave unstable terahertz nanonetworks. Computer Networks, 2020, 179, pp.107375 (12). hal-02993835

\section{HAL Id: hal-02993835 \\ https://hal.science/hal-02993835}

Submitted on 7 Nov 2020

HAL is a multi-disciplinary open access archive for the deposit and dissemination of scientific research documents, whether they are published or not. The documents may come from teaching and research institutions in France or abroad, or from public or private research centers.
L'archive ouverte pluridisciplinaire HAL, est destinée au dépôt et à la diffusion de documents scientifiques de niveau recherche, publiés ou non, émanant des établissements d'enseignement et de recherche français ou étrangers, des laboratoires publics ou privés. 


\title{
Efficient Routing Protocol for Concave Unstable Terahertz Nanonetworks
}

\author{
Lina Aliouat ${ }^{\mathrm{a}, *}$, Hakim Mabed ${ }^{\mathrm{a}}$, Julien Bourgeois ${ }^{\mathrm{a}}$ \\ ${ }^{a}$ University Bourgogne Franche-Comté FEMTO-ST Institute, CNRS, Montbéliard, France
}

\begin{abstract}
The recent progress in nanotechnologies is giving birth to a novel topology of wireless networks characterized by a high local density and an intensive node instability such as in WBAN and swarm micro-robots systems.

In this paper, we show that classical and dedicated ad hoc nanonetwork routing solutions are inefficient in this case and present a low reliability level and add a supplementary delay and control traffic. Majority of these solutions are based on point to point relaying mode, which is not adapted to the instability context.

The multirelay to multirelay approaches allow countering the problem of nanonodes uncertainty by using the high number of inter-node connections. However, these approaches perform badly when the nanonetwork deployment presents distortions and concave sides. We propose a new routing protocol called Multirelay to Multirelay Routing Protocol (M2MRPv2), which provides a natural way to manage the residual energy levels on the nanonodes. M2MRPv2 is, to the best of our knowledge, the only approach that proposes a proactive multirelay to multirelay routing mode where the residual energy level of the nanonodes and reliability of the routing paths are taken into account.
\end{abstract}

We study the performances of multirelay to multirelay protocols according to different multi-source to multi-sink communication scenarios. The obtained results show that M2MRPv2 protocol outperforms by far the Sustainable Longevity Routing (SLR) protocol (the reference protocol for Terahertz nanonetworks) in terms of transmission reliability and energy management. This outperformance is accentuated when the Terahertz nanonetwork deployment presents many concavities.

Keywords: Wireless nanonetwork, ultra-dense nanonetworks, unstable network, Terahertz band, concave topology, routing protocol, multirelay to multirelay routing, energy optimization.

\section{Introduction}

In this paper, we discuss the emergence of new radio networking architecture characterized by three features never gathered before. In these new systems, such as programmable matter and Wireless Body Area Network, (a) a huge number of nodes are concentrated in a narrow space with (b) uncontrolled distribution, and (c) are doted by a very limited but renewable energy capabilities. The objectives of this work are twofold. First, we give a detailed overview of the current capabilities of sub-millimeter nanonodes. Secondly, we show

\footnotetext{
* Corresponding author

Email addresses: lina.aliouat@univ-fcomte.fr (Lina Aliouat), hmabed@femto-st.fr (Hakim Mabed), julien.bourgeois@femto-st.fr (Julien Bourgeois)
} 
that both classical routing protocols and dedicated nanonetwork routing protocols are not adapted to such conditions. We propose then a new version of the M2MRP protocol [1] to overcome these constraints.

The advances in both Micro-Electro-Mechanical Systems (MEMS) and Terahertz telecommunication domains are giving birth to new ad hoc network topologies composed of a huge number of sub-millimetric nodes with unprecedented density. By comparison, Internet network involves up to billions of terminal nodes with an average connection degree of about 6 connections per node [2], while ultra-dense nanonetworks can present an average degree (number of neighboring nodes per node) higher than 1000 nodes. The submillimetric size of the devices leads to energetic and memory limitations, and the use of Terahertz band frequencies represent a real issue for data communication. Moreover, it is not always possible to control the nanonetwork's global shape that may present some concave sides. An example of the nanonetwork topology distortion is given in figure 1, where the nanonodes are deployed inside a human bowel. The nanonodes distribution is dictated by the bowel shape.

In this context, traditional routing protocols such as AODV [3], DSR [4] based on a chain of retransmissions (one node every time) are a risky strategy in terms of communication reliability. Major recent protocols dedicated to the nanonetworks such as $[5,6,7,8]$ also adopt the point to point relay mode. Besides, the node energy management consists in reducing the overall consumed energy without taking into account the residual energy on the nanonodes [6, 7]. In contrast, in multirelay to multirelay routing protocols, such as Flooding method [9], OLSR [10], and SLR [11], every node forwards received data to a subset of neighboring nodes. This way, communication reliability is improved since the data transmission does not depend on a single node. However, no multirelay to multirelay approach does deal with the energy aspect. Also, the shape of the nanonetwork topology is ignored, which makes difficult the adaptation of the multirelay to multirelay approaches such as CORONA [12] and SLR [11].

We proposed a new routing protocol called M2MRPv2; which is, to the best of our knowledge, the first proactive multirelay to multirelay routing protocol that takes into account the network topology and the residual energy of the nodes. We assessed the performances of our new protocol in terms of reliability and the number of exchanged messages in the context of ultra-dense unstable nanonetworks. We considered the Multi-source to Multi-Sink routing problem where nanonodes produce data that should be sent to the sink nodes representing gateways towards the decision unit. To this end, we simulated a Time Spread On-Off Keying protocol (TS-OOK) [13] based Terahertz nanonetwork system composed of sub-millimeter nodes and we simulated the energy and memory capacities of such nodes. Then we compared the performance of our approach with broadcast and SLR protocols.

The paper is organized as follows. The next section discusses some of the major routing protocols for the ad hoc network. Special attention is given to the recent protocols dedicated to nanonetwork systems. In section 3, we list the main contributions of this work. In section 4, we describe the multi-source to multi-sink routing problem and the Terahertz nanonetworks characteristics. Then we study the capabilities of sub-millimeter nodes in terms of memory, communication, and energy in light of current technological 


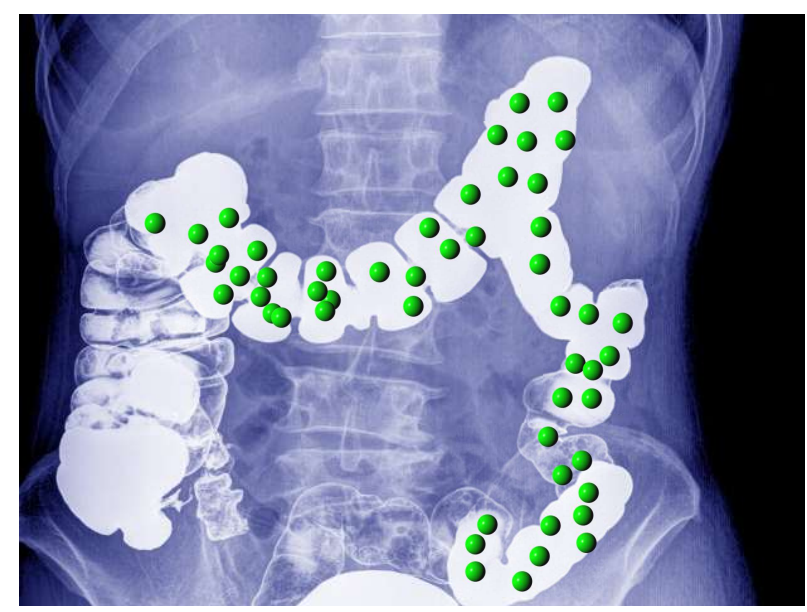

Figure 1: Wireless Body Area Network (WBAN) deployed inside a bowel. The nanonetwork deployment follows the bowel shape and consequently forms a concave shape.

advances. In section 5, we describe the new version of our M2MRPv2 protocol and in section 6 a complexity comparison between our method and well-known protocols is given. Section 7 is dedicated to experimental tests, followed by conclusions and perspectives.

\section{Related works}

The literature of routing protocols in ad hoc networks is very rich. However, only a few works deal with routing in ultra-dense nanonetworks with unstable radio links.

Some recent works investigate the problem of routing in nanonetworks $[14,15,16]$. However, those works deal with the energy and memory limitations by simplifying the routing procedure but do not address the nodes' instability issue. In [16], we present a 2.5 layer protocol that reduces the complexity (the density) of the nanonetwork. In this approach, the traffic regulation protocol smooths the traffic using TDMA scheduling and a selection of relay neighbors but the state of charge of the nodes is ignored. Other works, such as [17], provide some mechanisms to overcome node's volatility but the computational and spatial complexities of the method were ignored particularly in ultra-dense nanonetworks. Pierobon et al. [6] proposed an original routing protocol for wireless nanosensor in Terahertz Band (noted Pierobon's protocol hereafter). The routing protocol uses the harvesting capabilities of each nanonode to determine the best way to send the message to its nano-controller.

In this section, we study some of the major routing protocols for conventional ad hoc networks in order to discuss the possibility of using them in ultra-dense nanonetwork. All discussed classical approaches gave birth to improved versions. We focus, in this study, on the bases of each method, that correspond to the simplest version. We also discuss some special protocols proposed for nanonetwork and discuss their ability to manage the density and the instability of the network. 


\subsection{Classical ad hoc network routing protocols}

Routing protocols for ad hoc networks depend on the nature of the ad hoc network: MANET, VANET, WSN, etc. In Mobile [18] and Vehicular [19] Ad hoc Networks, the priority is given to managing the network variability (distances, positions) in a reactive way. While in Wireless Sensor Networks, the objective is to extend the network lifespan [20].

OLSR protocol [10] is a proactive multirelay protocol. Each node establishes a subset of neighboring nodes that will serve as relay nodes. The reduction of the relay sets allows reducing the number of exchanged messages for data broadcasting. The node relays a received message only if it is registered as a relay point of the sender.

DSR [4] is a reactive routing protocol that uses a deduction process for determining the network topology starting from the routing packet headers. When the source node has not an entry for the destination in the routing table, it sends a route request packet. Once an intermediate node receives the route request, it adds its ID to the request and forwards it. When the request reaches the destination node or an intermediate node with a route to the destination, the complete route is then transmitted in a route reply packet to the source node. However, several improvements have been proposed for DSR protocol [21, 22] involving an analysis of topological information. From the paths included in the data packets, nodes extract the sub-paths towards the intermediate nodes. Additional information on the network topology could be deduced by combining the different paths. Nodes could also use the paths obtained from their neighbors. These improvements aim to reduce the computational complexity of the protocol. However, the topological deductions increase the risk of propagating incorrect information.

AODV [3] is a reactive routing protocol based on point-to-point routing. Each node that receives a packet computes the next hop node using a routing table. As for DSR protocol, the node diffuses a route request (RREQ) in order to determine the path to the destination node. This request is diffused only if the node has no path to the destination or if the availability date of the route has expired. During the propagation of the path request, the intermediate nodes store the neighboring nodes that relayed the request in the routing table entry corresponding to the source node in order to establish the reverse path. Once the path request reaches the destination node, it sends a path response packet (RREP) and the intermediate nodes update the routing table entry corresponding to the destination node. Since the nodes update the routing tables according to reverse path principle, AODV cannot be used when the communication between nodes is asymmetric.

\subsection{Nanonetwork routing protocols}

CORONA protocol is a routing technique proposed in 2015 by Tsioliaridou et al. [12] and specifically targets ultra-dense nanonetworks. At the beginning of the protocol, each node computes its coordinates relatively to special nodes called anchors. Each anchor node $a_{i}$ broadcasts a setup packet that allows each node, $x$ to compute its coordinates, $x_{i}$ relatively to the anchor, $a_{i}$ as the minimum number of hops to reach it. 


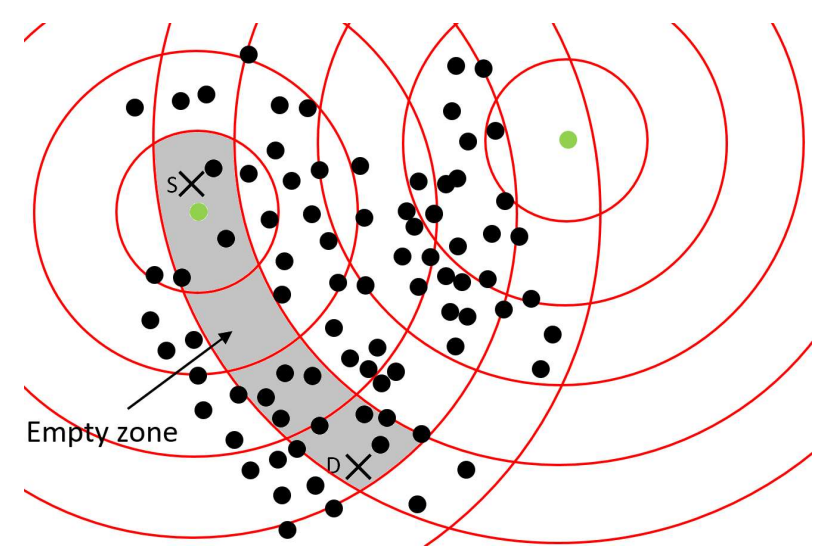

Figure 2: SLR functioning scheme. The coordinates system is computed according to the two anchor nodes (in green). The communication between source and destination nodes (cross marks) is not possible since the direct line includes an empty zone.

At the end, each node, $x$ knows its coordinates $\left(x_{1}, x_{2}, \ldots, x_{n}\right)$ where, $n$ is the number of anchor nodes. The network area is then subdivided into zones where each zone designates the nodes with the same coordinates (see figure 2).

During the data transmission, when a node $x$ receives a data packet, it checks if it belongs to the area delimited by the source, $s$ and destination, $d$ coordinates following the equation 1 . If it is the case, it relays the received data.

$$
\forall i ; x_{i} \in\left[\min \left(s_{i}, d_{i}\right), \max \left(s_{i}, d_{i}\right)\right]
$$

An improvement of the CORONA approach, called Stateless Linear-path Routing (SLR) has been proposed in [11]. In SLR protocol, the node forwards the received data packet if it belongs to the direct path between the source and the destination nodes as shown in figure 2. If so, it forwards the data packet otherwise it ignores the packet.

Pierobon's routing protocol [6] is a dedicated routing protocol for wireless nanosensor networks, where a set of nanosensors detect nanoscale events and send their measures to the nano-controller nodes. Each time a nanosensor asks for transmitting data to its nano-controller, the nano-controller checks either the direct transmission is better or the use of a multi-hop transmission (use of an intermediate node to relay the data). The decision is made according to the energy consumption estimation of each option. Pierobon's approach aims to reduce the overall transmission energy but does not take into account the residual energy at each node. On the other hand, the method assumes that the nodes are randomly distributed over the area with a constant density, $\rho$ making the method not adapted to concave deployment topologies. The method assumes that all nanonodes can communicate directly with the nano-controller; which represents a strong restriction about the use of the method: the need for numerous nano-controllers, a flat landscape without obstacles, etc. Finally, the method adopts a reactive routing mode where the transmission path is computed just in time, which induces some latency. This latency increases when the intermediate node refuses the reception or the 
re-transmission of the incoming data due to its own conditions such as its residual energy, buffer capacity, and SNR (Signal to Noise Ratio).

EECR protocol [7] proposes a cooperative protocol for hierarchical cluster-based nanonetworks. The nanonodes of a given cluster cooperate to forward data to the nano-controller (sink node). The nanonodes cooperation aims to determine the optimal path according to the energy cost. As with Pierobon et al. protocol, the objective is to reduce the amount of the total consumed energy without considering the residual energy level on each nanonode.

LaGOON [5] is one of the rare routing approaches for nanonetworks that take into account the nodes' volatility. LaGOON uses the destination MAC address of the received packet to identify the targeted relay and uses the network address to identify the final destination of the packet. In LaGOON protocol, when a node needs to send or relay data to a given destination node for which it has not a path, it floods the message to all its neighbors. Every node receiving a message from the node, $s$ relayed by the neighbor, $v$ uses it to establish or to update the route toward $s$ via $v$. If the destination MAC address of the received message is different from the receptor's own address and different from the broadcast address than the message is ignored. Otherwise, the message is relayed.

The objective of LaGOON protocol is to eliminate the need for control messages. The routes are built using the exchanged data packets. At the starting of the nanonetwork, data transmission follows a flooding pattern. Finally, the network nodes store for every sink destination the set (or subset) of next hops to reach it. The set of good neighbors is sorted in such a manner to maintain the last direct sender in top. Therefore, the use of the last sender node can locally concentrate the traffic on one node leading to collisions and energy over-consumption. Moreover, the radio link is assumed symmetric, which is not the case.

\section{Contributions}

This paper presents a first study of routing protocols efficiency for ultra-dense unstable wireless nanonetwork. Other studies provide a comparison between routing protocols in nanonetworks [23] and ad hoc networks [24], but the volatility of nodes (energy aspect) and the high density (memory and computing complexity aspects) are not taken into account.

Rather than assuming extreme capabilities situation (unlimited or nearly non-existent), we have studied the current possibilities offered to the sub-millimeter nodes in light of the current progresses of the technology. We discuss the energetic and memory capabilities of nanonodes and their impact on the feasibility and reliability of classical routing approaches (ad hoc and mobile ad hoc protocols). Furthermore, we present a critical analysis of the routing protocols dedicated to nanonetworks and highlight their lack of reliability and adaptability.

Special attention is given to multirelay routing approaches and a comparison of their performances is done to assess the way nodes' energy is managed. We introduce a new version of our multirelay routing protocol called Multirelay to Multirelay Routing Protocol (M2MRPv2). The approach represents the first 
multirelay to multirelay routing approach that takes into account the residual energy level of the nodes. We show the efficiency of M2MRPv2 compared to SLR and classical flooding methods. The tests are achieved on a set of nanonetwork scenarios presenting different network topologies, energy harvesting processes, and traffic patterns whilst major works in this field assume naive CBR traffic [25] with homogeneous topology.

\section{Routing problem in Terahertz nanonetwork}

A Terahertz nanonetwork is an ad hoc wireless network where the communication between sub-millimetric nodes is carried by sub-millimeter radio waves. The choice of sub-millimeter radio waves is dictated by the use of sub-millimetric antennas. The nature of targeted applications leads to a high density of nanonodes contemplated through technologies such as Graphene antenna [26] and MEMS components [27]. Due to the Terahertz radio signal and the energy capabilities of the nanonodes, the signal range is very short (signal power of less than $1 \mathrm{mw}$ covering about dozens of centimeters).

Furthermore, we study here kind of applications where a high number of sub-millimetric nodes are deployed in a narrow area (about $1 \mathrm{~m}$ of large). Such applications include programmable material [28, 29], Internet of Nano-Things [30], wireless nanosensor network WNSN [31, 32]. It should be noted that the density issue is also raised in bigger global mobile networks [33] such as 5G networks.

\subsection{Multiple sink problem}

Routing in nanosensor networks is a very common problem [32,34]. The nanosensors measure environmental conditions such as temperature or pressure and forward the data to particular nodes called sinks. We assume that sink nodes are not affected by the volatility phenomena and represent gateways to the decision unit.

\subsection{Access protocol in Terahertz nanonetwork}

Even if the presented study applies to any wireless radio access technique, we considered here the TS-OOK [13] protocol. TS-OOK is an On-Off keying protocol. Periodical symbols corresponding to electromagnetic pulses of 100 femtoseconds or silences are used to code respectively the 1 and 0 symbols. The duration between two consecutive symbols is fixed for all communications or notified by the sender during the communication announcement on the control channel [35].

\subsection{Analysis of sub-millimeter machine}

Sub-millimeter node takes different states during its operating cycle according to the current task: transmission, reception, idle, sleep, etc. Each step presents a specific energy increasing or decreasing process. The characterization of the nanonetwork requires the specification of the amount of energy required by each phase. 


\subsubsection{Communications and energy consumption}

Many modeling studies were made for characterizing radio communication energy consumption [36, 37]. TS-OOK specifications estimate the energy required by a pulse to $E_{p}=100 \mathrm{aJ}=10^{-16} \mathrm{~J}$. However, the diffused energy of the pulse represents just a part of the energy needed to transmit a "1" symbol, $E_{s}$, that includes a power amplifier, Frequency synthesizer, etc. We notice $E_{f}^{t x}$ the average energy required for transmitting a data frame of $n$ symbols $(0 / 1)$.

$$
E_{f}^{t x} \leq n \times E_{s}
$$

On the other side, data reception consumes energy less than energy required for transmitting data. This energy is used by the receiver components such as Low-noise amplifier (LNA). We notice $E_{f}^{r x}$ the energy required for the reception of a frame of $n$ symbols.

$$
E_{f}^{r x}<E_{f}^{t x}
$$

In addition to the communication task, nanonodes consume energy during their normal activity state corresponding to listening task, electronic components activities, sensing tasks, etc. The energy consumption during activity state varies over time according to the nature of performed tasks. Let $E^{a c}$ the average energy consumption per second due to the nanonode activities. It is also possible to envisage a sleeping mode that allows nanonodes to reduce their activities and, consequently, the energy consumption. We notice $E^{s l}$ the energy consumed per second during sleeping mode.

\subsubsection{Battery features at sub-millimeter scale}

The energy limitation of the nanonode is mainly dictated by the node size and, consequently, the battery size. Let $d_{n}$ the volume of a node and let $d_{b}$ the space consecrated to the battery $\left(d_{b}<d_{n}\right)$. The maximal amount of energy stored by a battery, $E_{\text {max }}^{b t}$, depends on the manufacturing materials of the battery. For example, a Lithium-Ion battery presents a density of energy of 0.36 to $0.95 \mathrm{~J} / \mathrm{g}$. Knowing that the volumetric mass of the Lithium-Ion battery is $0.2 \mathrm{~kg} / \mathrm{l}$. We deduce that within $d_{b} m^{3}$, the energy amount of a battery is equal to:

$$
E_{\max }^{b t}=\text { volumetric mass } \times \text { density of energy } \times d_{b}
$$

If the Lithium-Ion battery is of $0.2 \mathrm{~m}^{3}$, the maximum energy is of $14 \times 10^{-6} \mathrm{~J}$ to $38 \times 10^{-6} \mathrm{~J}$

Without harvesting mechanisms and if we ignore the other tasks of the nanonode, a Lithium-Ion battery of $0.2 \mathrm{~m}^{3}$ size and $E_{\max }^{b t}=20 \times 10^{-6} \mathrm{~J}$ can transmit up to $2 \times 10^{11}$ pulses. If the energy per "1" symbol is

of $E_{s}=10^{-15} \mathrm{~J}$. The battery could support about $10^{12}$ frames of 1000 symbols. However, by taking into account the other tasks of the nanonode, the required throughput of the application, the energy consumed by receiving, and relaying frames coming from thousands of other nodes, we deduce that the battery capacity is a very critical resource that should be managed with great attention. 


\subsubsection{Memory capacity at sub-millimeter scale}

In terms of memory capacity of the sub-millimeter node, the current advances allow expecting a memory size of about a few dozens of MB [38]. On the other hand, the memory size impacts on the energy consumption of the nanonode (activation, pre-charging, refreshing, read, write) [39]. The consumption level of each of these tasks depends on the type of memory (DRAM, SRAM, etc.). Nanonetwork applications should limit the memory requirements. Indeed, refreshing energy, that allows keeping the data stored in the memory, is proportional to the used memory. For example, Crucial DDR4-2133 memory model presents a consumption level at the idle mode of $3.5 \times 10^{-3} \mathrm{~J} / \mathrm{s}$ for a memory of 10MB. An energy level high enough compared to the energy provided by the nano-battery. Consequently, even though that memory of $10 \mathrm{MB}$ is currently possible in a sub-millimeter node, the memory size that could be supported by the battery is well below. One of the direct consequences of that is the impossibility of using some networking protocols that require high memory storage.

\subsubsection{Energy harvesting}

Due to the constrained limitation of the nano-battery, many works pay attention to the ways to recharge the battery while the node operates [40]. The harvesting mechanism is based on a power management device that stores a part of the energy provided by an external system to supply power to the node. In [41], the authors classify the harvesting techniques according to the energy source into mechanical, thermal, radiant, and biochemical sources. For example, in [40], a solar-based harvesting system is studied while in [42], the electromagnetic-based system is proposed. Whatever the used harvesting system, the nodes are able to restore, over time, a part of its energy. The amount of restored energy in a time unit depends on the nature of the harvesting system (in the solar-based system it may be impacted by the brightness intensity) and the battery characteristics. Boisseau et al. [41] estimates that the restoration of 10 to $100 \times 10^{-} 9 \mathrm{~W}\left(10^{-9} \mathrm{~J} / \mathrm{s}\right)$ is a good order of magnitude for energy harvester of about $1 \mathrm{~mm}^{3}$. Let $H(n)$ the recursive incremental function that returns the battery level on the node $n$ after one time unit.

$$
H(n)=H(n)+\delta(n)
$$

$\delta(n)$ designates the amount of harvested energy during the last time unit.

\subsection{Traffic characteristics in nanonetwork}

The study of the routing protocols performance needs to identify in advance the targeted application types. The nature of the application determines the probabilistic model of the occurring events. Exhaustive identification of these applications represents a tremendous work and is still somewhat premature. However, we distinguished in our work two case studies. The first case assumes that the nodes are independent and each node is regularly a source of data packets. This case fits well with sensor networks case, where every sensor (node) senses the environment and diffuses the measures. In the second case, we consider that 


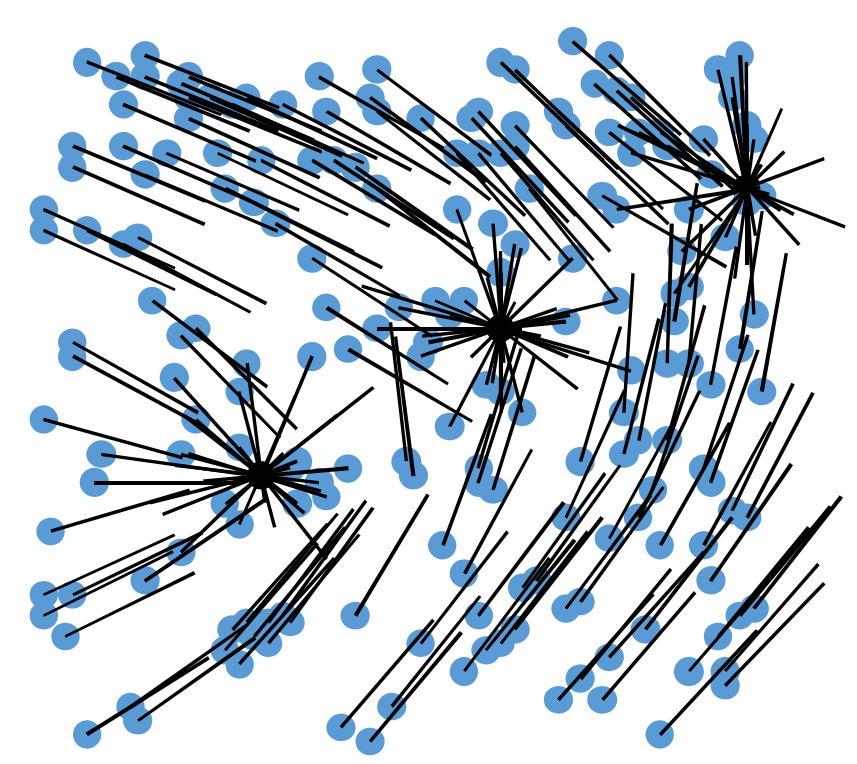

Figure 3: An example of M2MRPv2 data flows pattern describing the trajectory of data from any source node towards one of the three available sink nodes.

occurring traffic leads to relatively simultaneous data packets sent by the nodes near the event location. This case well represents positioning and tracking applications, where the nodes close to the foreign object detect and help to compute the position of the object.

\section{Improved Multipoint-to-Multipoint Protocol (M2MRPv2)}

M2MRP protocol is a new routing protocol that we proposed in [1]. We give, here, a new version called M2MRPv2 that adopts a new way to compute the main orientation of the nodes antenna (equation 6).

The idea of the M2MRPv2 protocol is to bring out a communication flow pattern defining the way the data are transferred from any ordinary nanonode to one of the sink nodes as shown in figure 3. So the flows' circulation structure corresponds to a distributed data structure that stores, in every ordinary node, the steering directions for receiving and sending data. The nanonodes are assumed to use directional antennas $[43,44]$.

For that end, M2MRPv2 draws on electrostatic physics to determine how the nanonodes compute the best direction (and not the best path) to reach one of the sink nodes. The protocol is based on three essential principles:

- Each node exerts an attraction force on the nodes within a given range.

- The attraction force of a node, $a$ on a node, $b$ depends on the received signal power from $a$ at $b$.

- The attractiveness vector of a given node is a result of the attractiveness vectors of its direct neighbors.

We conclude that the attractiveness vector of a given node is obtained from the combination of the attractiveness vectors of its direct neighbors. At the same time, the attractiveness vector of a given node affects 


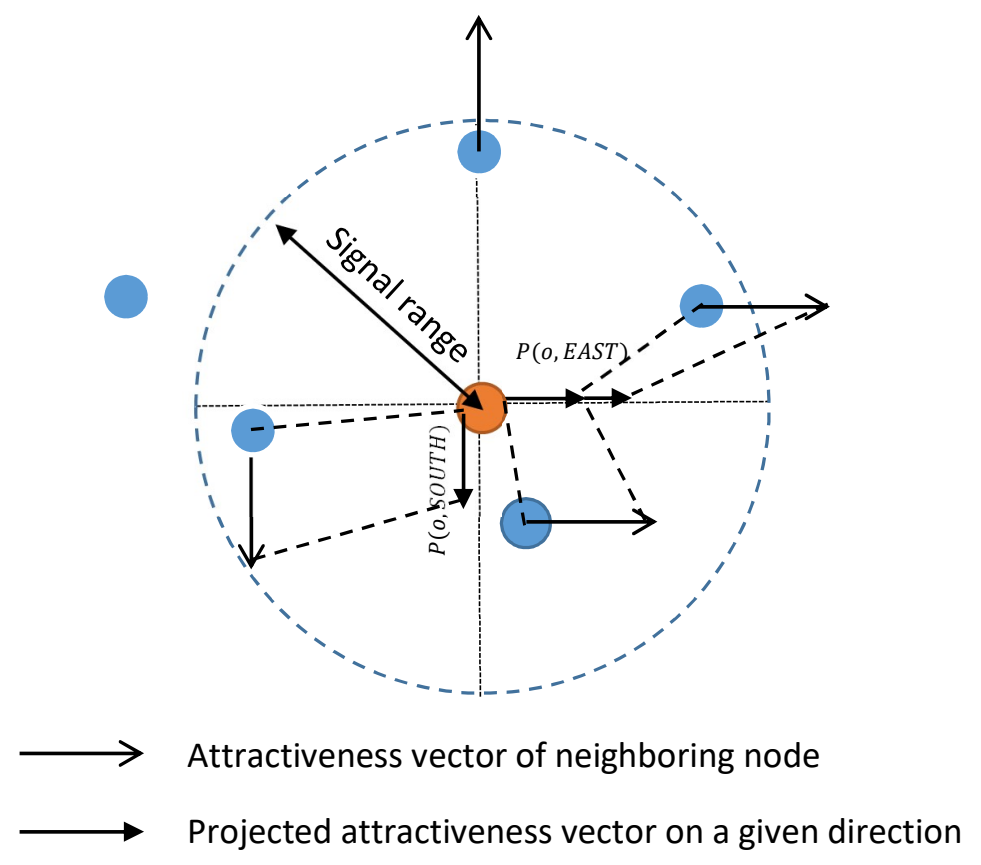

Figure 4: The computing of the attractiveness vector of the reference node in the center. For simplification, only cardinal directions are considered. The computation of $P(o, E A S T)$ and $P(o, S O U T H)$ are given. $P(o, W E S T)$ and $P(o, N O R T H)$ are equal to zero. The East direction (the best direction) is selected as the attractiveness vector. The node located in the extreme West is ignored since it is out of coverage of the reference node.

the attractiveness vectors of its neighbors. Consequently, each node affects indirectly the attractiveness vectors of farther nodes (propagation effect). The periodical distributed updating of the nodes' attractiveness vectors results in the formation of circulation lines, that represent the data circulation flow from ordinary nanonodes to the sink nodes.

Every $0.1 \mathrm{~ms}$ and in asynchronous way, each node sends its attractiveness vector in all directions and every $100 \mathrm{~ms}$, each node $o$ updates its attractiveness vectors $\overrightarrow{d(o)}$ using the received neighbors attractiveness vectors. Let $V(o)$ the set of neighboring nodes of $o$. We notice pow $(v, o)$ the power of the received signal from the node $v$ on the node $o$. Let $\overrightarrow{d(v)}$ the attractiveness vector of the node $v$ sent in the last control packet of the node $v$.

The attractiveness vector of the node $o$ is then updated according to the expression 6 . An illustration of the attractiveness vector calculation is also given in figure 4 .

$$
\begin{aligned}
& \overrightarrow{d(o)}=\{\overrightarrow{d *} ; \forall \vec{d}: P(o, \overrightarrow{d *}) \geq P(o, \vec{d})\} \\
& \text { with : } \\
& P(o, \vec{d})=\sum^{v \in V(o)} \operatorname{pow}(v, o) \times \max (0, \cos (\vec{d}, \overrightarrow{d(v)})) \times E(v)
\end{aligned}
$$

Sink nodes, $s \in S$, present a specific case where the sent attractiveness vector $\overrightarrow{d(s)}=(\infty, \infty, \infty)$. When an ordinary node $o$ receives one or several control packets with attractiveness vector $(\infty, \infty, \infty)$, it sets its 


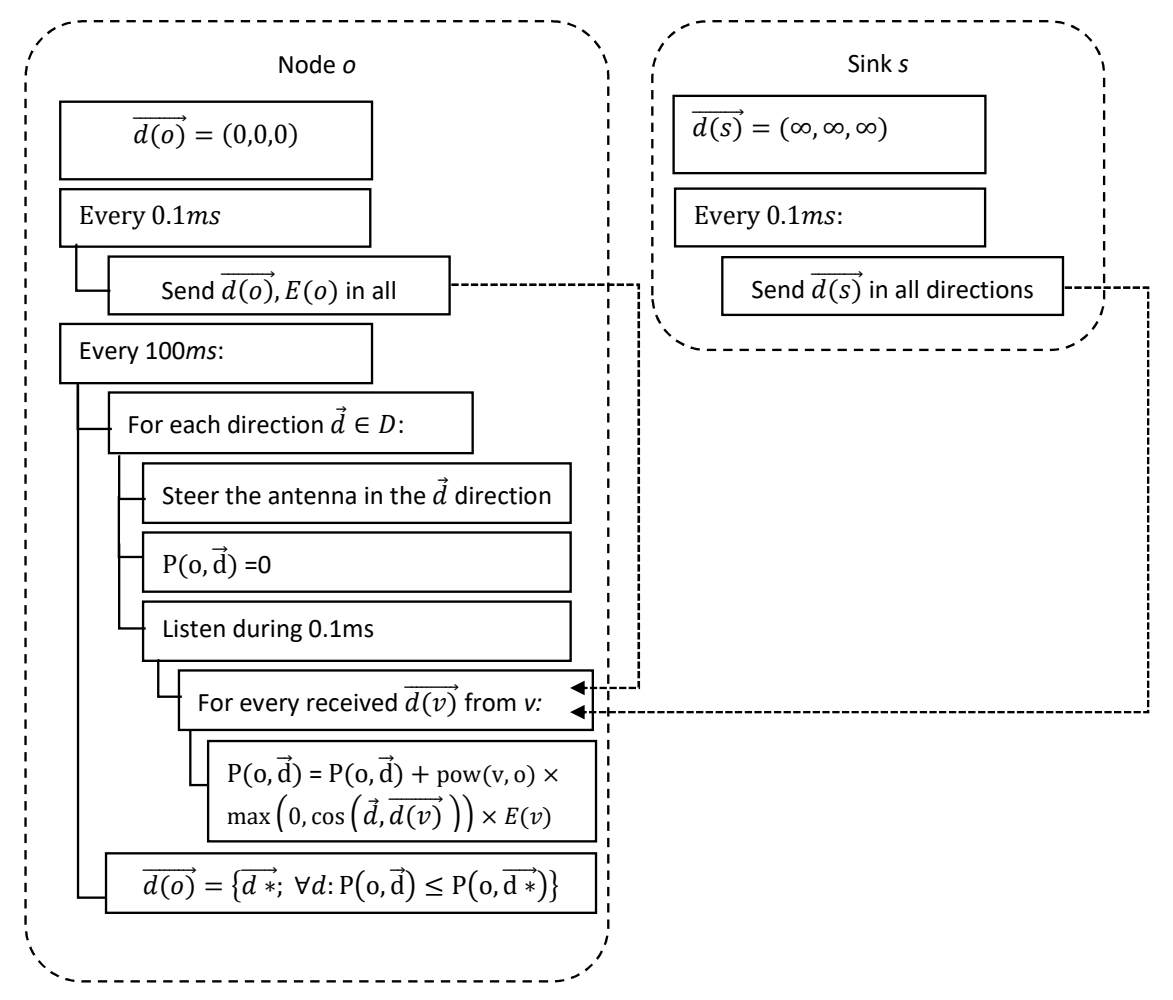

Figure 5: Block Scheme of the multirelay to multirelay routing protocol including an ordinary nanonode $o$ and a sink node $s$.

own attractiveness vector in the direction of the sink node (current direction $d$ ) and ignores the equation 6. The functioning scheme of the protocol is summarized in figure 5.

M2MRPv2 shares the same global scheme as M2MRP. For more details, readers may refer to the paper [1]. The computation of the attractiveness vector represents the key characteristic of the algorithm. In the precedent version, the attractiveness vector is computed using the equation 7 :

$$
\begin{aligned}
& \overrightarrow{d(o)}=\{\overrightarrow{d *} ; \forall \vec{d}: P(o, \overrightarrow{d *}) \geq P(o, \vec{d})\} \\
& \text { with : } \\
& P(o, \vec{d})=\sum^{v \in V(o)} \operatorname{pow}(v, o) \times \cos (\vec{d}, \overrightarrow{d(v)})
\end{aligned}
$$

The main difference between equations 6 and 7 consists in the computation of the weight of each direction $P(o, \vec{d})$ in order to determine the best one. In the new version (equation 6 ), the neighbors with a negative contribution regarding the direction $\vec{d}$ are ignored in the computation of $P(o, \vec{d})$. To illustrate the impact of this change on the behavior of the method, let us take the example illustrated in figure 6 . The reference node $o$, in the center, computes the $P(o, \vec{d})$ for each possible direction. We assume, for the explanation, that the set of possible directions is $D=\{N, S, E, W\}$ corresponding to the four cardinal directions. The node $o$ is surrounded by a set of 13 nodes, 6 on the East side, 6 on the West side and one node on the North side. The nodes on the East and West sides present a perfect symmetry and attractiveness vectors received by $o$ from the 12 nodes offset one another. Using the equation 7 of the precedent algorithm version 


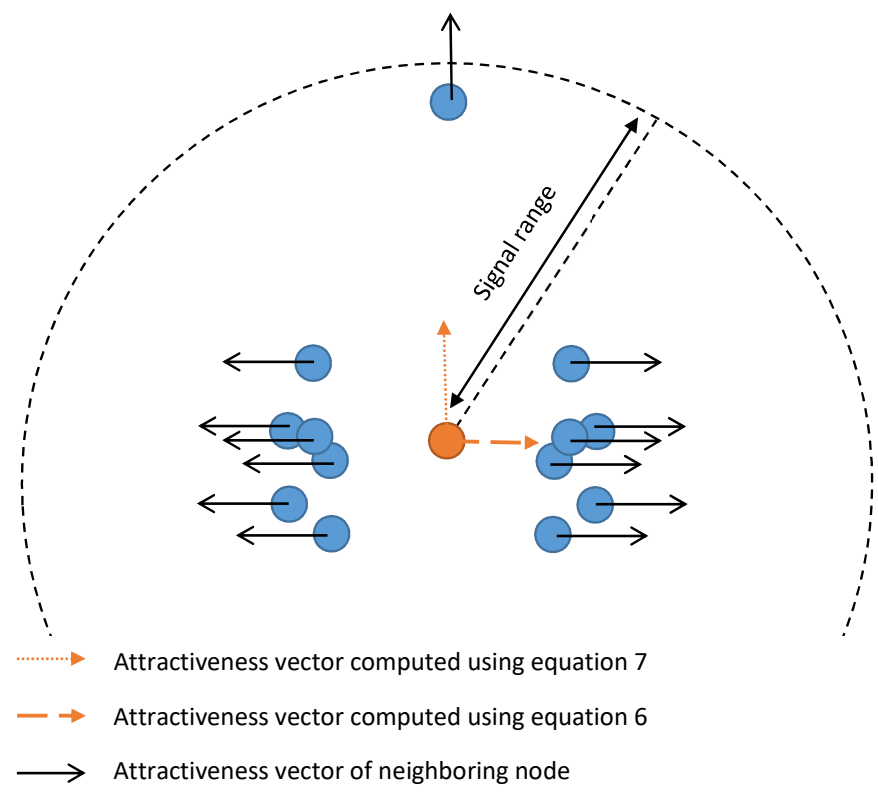

Figure 6: Comparison between the computation of the attractiveness vector in M2MRP and M2MRPv2 methods. Reference node is in the center.

M2MRP, the attractiveness of the East direction, $P(o, E A S T)$, and the West direction, $P(o, W E S T)$ are null. Therefore, the North direction will be selected even if there is only one node on the North side, which is far from $o$. In contrast, with equation 6 , the computation of $P(o, E A S T)$ ignores the nodes on the West side of $o$ and vice versa for $P(o, W E S T)$. Consequently, in M2MRPv2, either the West or the East direction would be selected, which increases the robustness of the method.

\section{Comparison of routing protocols}

In this section, we present a comparative study of the protocols presented in the related works section and our M2MRPv2 protocol. This comparison is made according to the following criteria:

- The space complexity that represents the amount of memory space required by the protocol.

- The routing rapidity that designates the required transfer time from the source node to one of the sink nodes.

- The number of sent messages that designates the number of sent packets generated for routing one packet from a source node. This number impacts on the quality of energy management.

- The number of received packets due to the transmission of one packet from a source node. 
- The number of control messages evaluates the number of exchanged messages needed to determine the routing path of the current packet.

- The need for checking the source of the packet before relay it.

- The need for checking the destination of the packet before relay it.

Table 1 gives a comparison of the 8 discussed methods according to the 7 criteria listed above, where $|V|$ is the average number of neighbors, $S$ is the set of sinks, $|E|$ is the number of pairs of neighbor nodes, and $|D|$ represents the number of steering directions of the antenna. We observe that M2MRPv2 method is the only protocol that does not need control messages, nor checking the source or the destination of the packets, which reduces the routing latency. We observe also that only M2MRPv2 selects the routing path on the basis of the density of the crossed areas, which contributes to the reliability of the protocol.

The complexity of AODV and LaGOON presents a lot of similarities. Though contrary to AODV, LaGOON protocol eliminates the need for control messages. If the route is not known, the message is diffused to all direct neighbors using omnidirectional or directional signals. If the node's antenna is omnidirectional then $|D|=1$. LaGOON presents higher memory complexity than AODV since a set of next hop nodes are stored for each sink. In ultra-dense nanonetwork, the set of stored next hops is more probably equal to the set of neighbors. Pierobon and EECR protocols present good memory complexity since they are proposed for nanonetworks. However, these two approaches compute the transmission path in real-time which leads to additional control traffic and more latency.

Concerning the multirelay to multirelay approaches, even if the flooding approach ensures the fastest transmission of data but it leads to a high amount of exchanged messages and consequently a high level of energy consumption and interference. M2MRPv2 and SLR generate less exchanged messages than the flooding method but the efficiency of SLR method depends on the average density of a zone $Z$. Dense zones can lead to a high level of exchanged messages while blank zones can lead to communication failures (see figure 2).

In table 2, we compare the different features of routing protocols proposed for nanonetworks. Among all the algorithms, we observe that only our protocol M2MRPv2 allies the use of the multirelay to multirelay mode with the optimization of the residual energy level of nanonodes. Both features contribute to the improvement of communication reliability.

\section{Experimental tests}

In this section, we focus only on the studied multirelay protocols, since Point-to-Point protocols are ineffective for unstable networks. The OSLR protocol is ignored because it is very demanding for memory space $\left(\mathrm{O}\left(|V|^{2}\right)\right)$. Three routing protocols are then compared, corresponding to the naive broadcasting approach, SLR protocol, and M2MRP protocol. The three approaches are compared on two network scenarios representing square and tie bow topologies with 100, 300, 500, 700, and 1000 nanonodes among which three sink 


\begin{tabular}{|l|c|c|c|c|c|c|c|}
\hline Method & $\mathrm{O}(\mathrm{mem})$ & $\mathrm{O}(\mathrm{t})$ & \#sent mess & \#rcv mess & \#ctrl mess & check src & check dst \\
\hline AODV & $|S|$ & shortest & $\mathrm{r}$ & $\mathrm{r}$ & upto $\mathrm{E}$ & & $\mathrm{x}$ \\
OLSR & $|V|^{2}$ & $>$ shortest & $<|D| \times N$ & $<|V| \times N$ & 0 & $\mathrm{x}$ & $\mathrm{x}$ \\
Flooding & 1 & shortest & $|D| \times(N-S)$ & $|V| \times N$ & 0 & $\mathrm{x}$ & \\
\hline Pierobon & 1 & lowest energy & $<r$ & $<r$ & $<2 \times r$ & $\mathrm{x}$ & $\mathrm{x}$ \\
EECR & 1 & lowest energy & $<r$ & $<r$ & $<2 \times r$ & $\mathrm{x}$ & $\mathrm{x}$ \\
LaGOON & $<|S| \times|V|$ & shortest & {$[r . .|D| \times(N-|S|)]$} & {$[r . .|V| \times N]$} & 0 & $\mathrm{x}$ & $\mathrm{x}$ \\
SLR & 1 & direct & $Z \times r$ & $Z \times 4 \times r$ & 0 & $\mathrm{x}$ & $\mathrm{x}$ \\
M2MRPv2 & 1 & denser & $\ll N$ & $\ll|V| \times N$ & 0 & & \\
\hline
\end{tabular}

Table 1: Complexity comparison between routing techniques for ad hoc networks and nanonetworks.

\begin{tabular}{|l|l|l|l|l|}
\hline Method & mode & Energy saving & Reliability & constraints \\
\hline Pierobon & reactive & total consumed energy & point to point relay & $\begin{array}{l}\text { - homogene topology } \\
\text { - direct link between } \\
\text { nanonodes and sinks }\end{array}$ \\
\hline EECR & reactive & total consumed energy & point to point relay & clusters computation \\
\hline LaGooN & reactive & - & point to point relay & symmetric links \\
\hline Flooding & reactive & - & multirelay to multirelay & - \\
\hline SLR & reactive & - & multirelay to multirelay & convex topology \\
\hline M2MRPv2 & proactive & residual energy & multirelay to multirelay & - \\
\hline
\end{tabular}

Table 2: Comparison between nanonetworks routing protocols.

nodes (see figure 7). The nodes are deployed over an area of $100 \mathrm{~mm} \times 100 \mathrm{~mm}$ and the radio signal range is $20 \mathrm{~mm}$, which guarantees the high density of the network. For each network scenario, we tested two traffic profiles representing both independent events and location-based events.

Finally, we tested several traffic load levels to analyze the evolution of the performances when the traffic increases. The traffic load, Lo, designates the average number of packets generated per node per communication cycle of $0.1 \mathrm{~ms}$. We tested 5 different values of the traffic load $0.1,0.2,0.3,0.4$ and 0.5 . In independent events scenarios, the number of packets generated by a given node during a given communication cycle is computed using the formula: $-\log (r n d() * \operatorname{Lo}$ where $r n d()$ is the function that returns a random value in [0..1]. In location-based events scenarios, at each communication cycle, a sequence of several random positions are selected. For each random event location, the nanonodes that are less than $5 \mathrm{~cm}$ from it, generate each a packet. The selection of event location process is stopped once the average number of packets per communication cycle is reached. One packet contains 1000 bits, and once a packet is generated, the packet routing is simulated within the same cycle. 


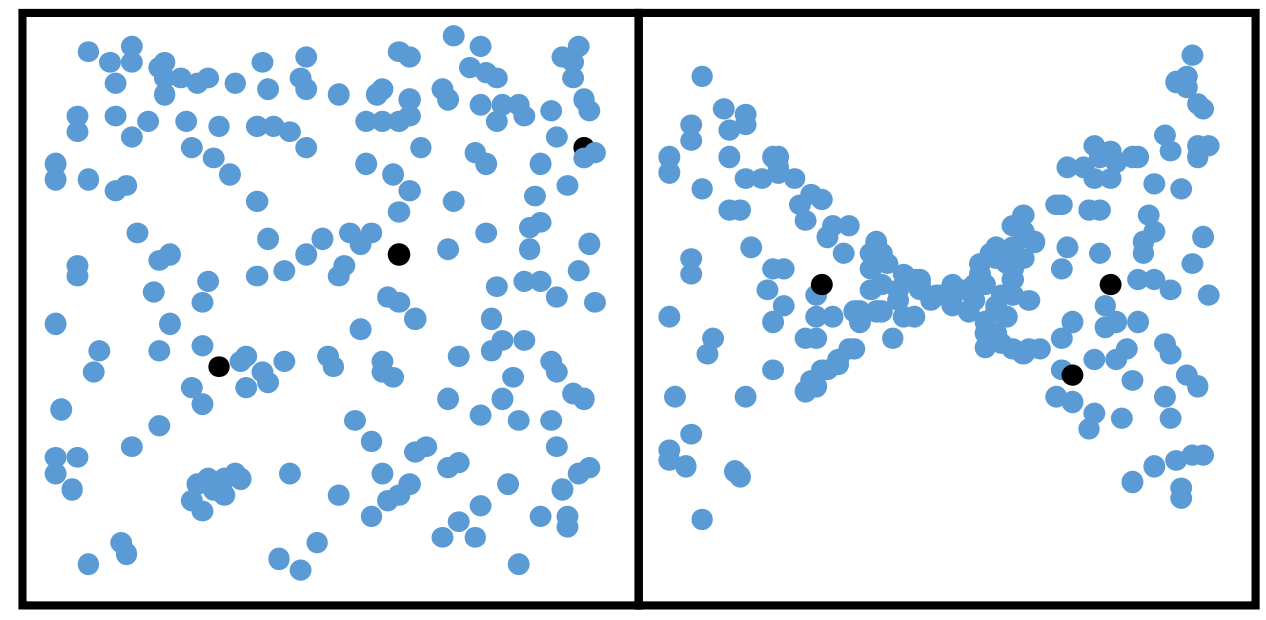

Figure 7: Two classes of nanonetwork topologies: square and tie bow. Black nodes represent sink nodes.

We note that collisions between received packets are not simulated in this work. The parameter settings used in the simulations are summarized in table 3.

The simulated traffic (chronology and source of packets) is the same in all scenarios with the same topology, and the same traffic profile and load. This way, we guarantee that protocols are tested under the same conditions. The scenarios use 3 sink nodes corresponding to the 10th, 20th, and 30th nodes. To facilitate the reproduction of these scenarios, readers can use the Microsoft Excel VBA source code presented in https://cloud.femto-st.fr/nextcloud/index.php/s/qzi8m2fRBW2NRRz and just set the number of nanonodes.

\subsection{Performance comparison}

The performance of each approach is assessed according to the number of sent messages (both generated and forwarded packets) and according to the success rate. The success rate measures the percentage of generated messages (from the source) that reached at least one of the sink nodes.

In figure 8, we present the obtained results. The left column compares the three approaches according to their success rate. First, we observe that M2MRP protocol outperforms the two other approaches in all cases, especially when the generated traffic by nanonode is low. This is explained by the fact that the M2MRPv2 protocol succeeds in sharing the relaying effort on all nodes. But when the generated traffic per node exceeds the harvesting rate, the transmission of data towards the sink nodes fails from the start. In this case, the routing strategies cannot provide a solution.

SLR performs slightly better than the naive broadcast method. However, the number of needed messages is significantly reduced by SLR method. We observe that for tie bow topology, the broadcast approach presents a better success rate when the energetic constraints are weak (low traffic). Indeed, SLR uses the direct line between the source and sink nodes, which makes it less reliable than the broadcast method when the energy limitation is not a problem. At the same time, when the traffic load increases, SLR reduces the 


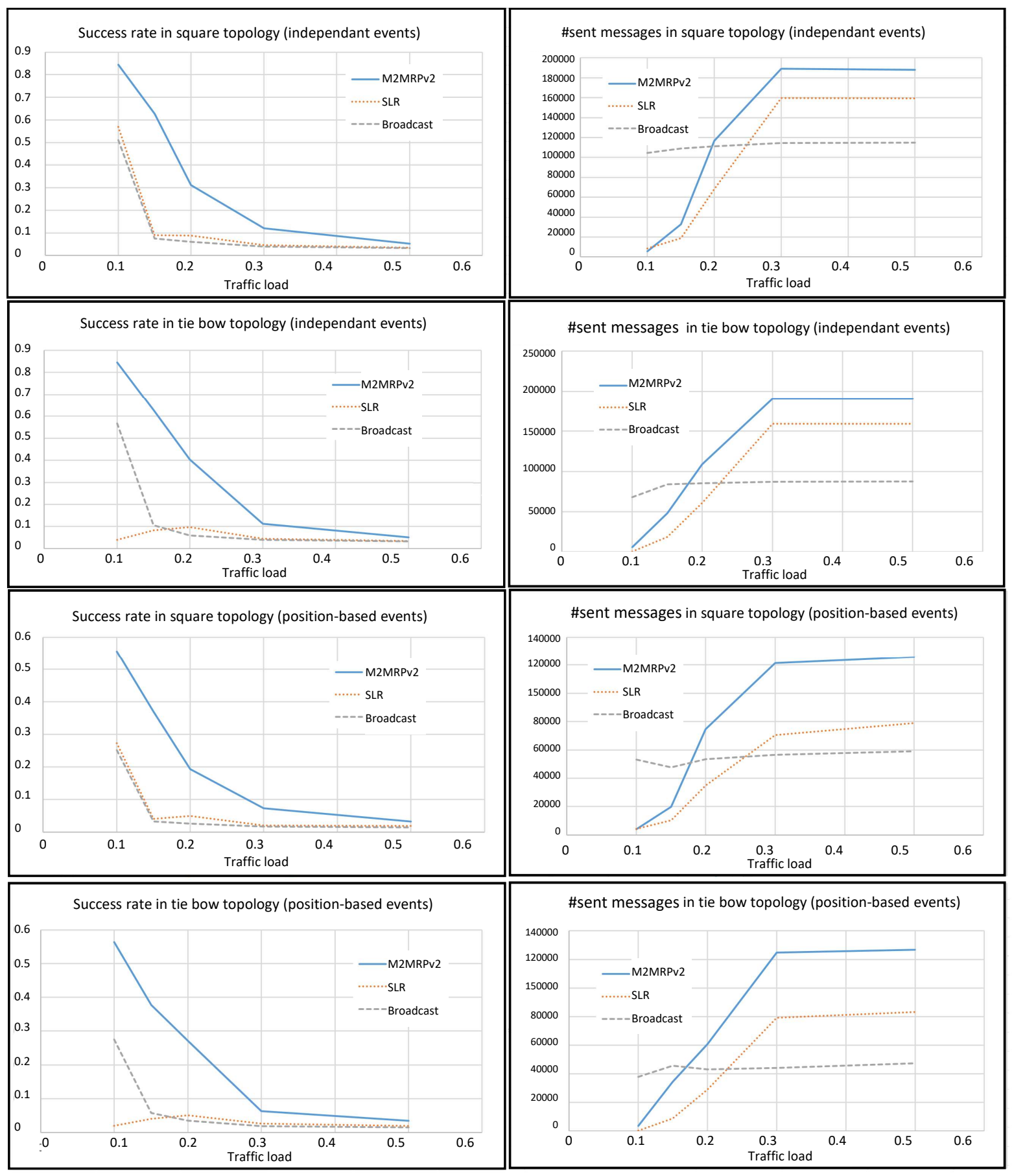

Figure 8: Comparison of broadcast, SLR, and M2MRPv2 routing protocols over two network topologies and under two traffic profiles. A comparison is given according to the success rate and the number of sent messages. The traffic load measures the average number of new packets generated each $0.1 \mathrm{~ms}$. 


\begin{tabular}{|l|l|}
\hline Parameter & Value \\
\hline Maximal stored energy: $E_{m a x}^{b t}$ & $100 \mu \mathrm{J}$ \\
Energy consumption for one packet transmission: $E_{f}^{t x}$ & $0.05 \mu \mathrm{J}$ \\
Energy consumption for one packet reception: $E_{f}^{r x}$ & $0.005 \mu \mathrm{J}$ \\
Energy harvesting rate per 0.1 sec: $\delta(0.1 \mathrm{sec})$ & $0.05 \mu \mathrm{J}$ \\
Energy consumption by nanonode activities or inactivity: $E^{a c}+E^{s l}$ & $0 \mathrm{~J} / \mathrm{s}$ \\
Communication cycle duration & $0.1 \mathrm{~ms}$ \\
Routing cycle duration (frequency of attractiveness vector computing) & $100 \mathrm{~ms}$ \\
Packet size & $1000 \mathrm{bits}$ \\
Number of simulated routing cycles & $1000(100 \mathrm{~s})$ \\
Radio range & $20 \mathrm{~mm}$ \\
Network size & $100 \mathrm{~mm} \times 100 \mathrm{~mm}$ \\
Number of sink nodes & 3 \\
\hline Hardware and software environment of simulation & \\
\hline Processor: Intel Core i7-7820HQ CPU $2.90 \mathrm{GHz}$ & \\
Processor architecture: 64 -bit & \\
RAM size: 16 GBytes & \\
Operating System: Microsoft Windows 10 & \\
Operating system core: 64 bits & \\
Programming environment: Microsoft VBA Excel professional plus 2016 \\
\hline
\end{tabular}

Table 3: Simulation parameters

number of sent messages and therefore manages better the energy limitations of nanonodes, which leads to better reliability.

In applications with position-based events, the efficiency of all protocols decreases. The traffic is less well distributed over the network nanonodes and generates a temporal peak of traffic on localized parts of the nanonetwork. However, the M2MRPv2 protocol is much better than SLR and broadcast approaches.

The number of sent messages shown in figure 8 is higher in M2MRPv2 protocol for overloaded networks. That does not mean that M2MRP generates more redundancy, just that the energy is better managed leading to better traffic spreading and therefore to more successful transmissions (i.e. more packet forwarding). Whereas in Broadcast and respectively in SLR, all or respectively some nodes are overused and can no longer relay the data. In other words, even if the multi-received packets are relayed just one time, the multireception of the same data impacts the state of charge of the nodes causing their temporal unavailability and then transmission failure. Consequently, under moderate traffic ( 0.1 packet per node per $0.1 \mathrm{~ms})$, the exchanged traffic remains high in the broadcast approach due to the network density and the number of 


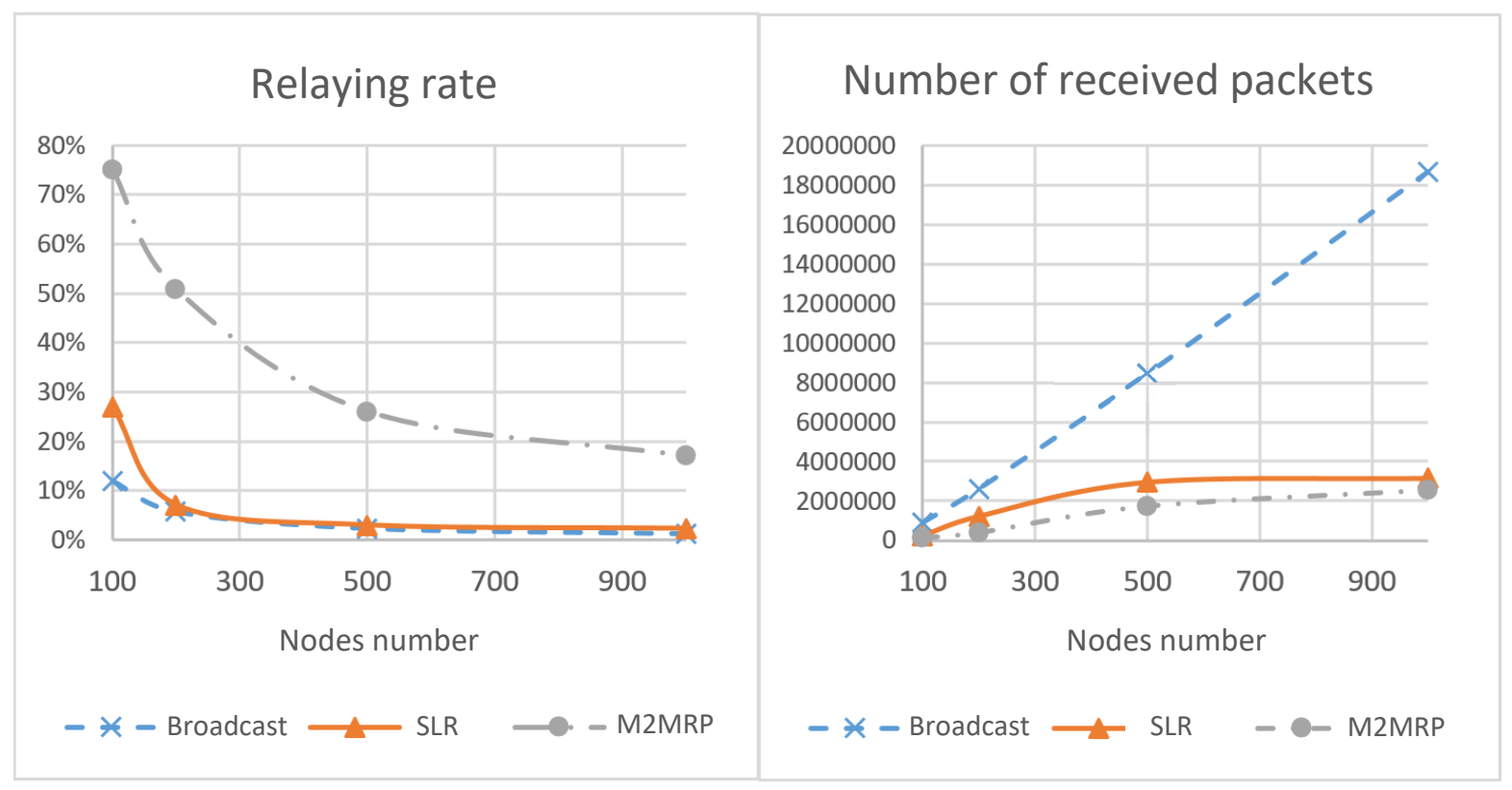

Figure 9: Comparison between Broadcast, SLR, and M2MRP protocols according to the number of received messages and relaying rate. Test parameters are the same as above and traffic load is of 0.2 packets per communication cycle per node.

re-transmissions and receptions of the packet. In the broadcast method, the received packets are relayed by all nodes, and nodes exhaust rapidly their battery leading to temporary unavailability. Broadcast method hits rapidly saturation, which results in the observed stagnation of the number of sent messages when the traffic load increases. SLR and M2MRPv2 approaches provide more sent packets. Indeed, SLR and M2MRP provide a better success rate, which means that packets are relayed farther than in broadcast in order to reach the sink nodes. The difference between the sent messages and received messages is given in figure 9 .

Figure 9, displays the percentage of relayed packets among the received ones, as well as the number of receptions over all nodes for each method. The relaying rate is a good indicator of the efficiency of the routing policy. A low value means that the protocol leads to a high level of redundancy with a lot of received packets that have already been received or that should be ignored (For example in SLR, according to the source and the destination nodes). We observe then that M2MRPv2 leads to extremely higher efficiency compared to SLR and broadcast methods with $51 \%$ of received messages that are retransmitted with 200 nodes and $75 \%$ with 100 nodes. This can be explained by the use of beam steering devices in M2MRPv2 protocol, which improves the targeting of the next hop nodes. Furthermore, the number of received messages in M2MRPv2 is significantly lower than in SLR and Broadcast protocols. We conclude that the performances of M2MRPv2 could be even better than those presented in figure 8 if the ratio between energy consumption for reception and emission is higher ( $10 \%$ in the test). 

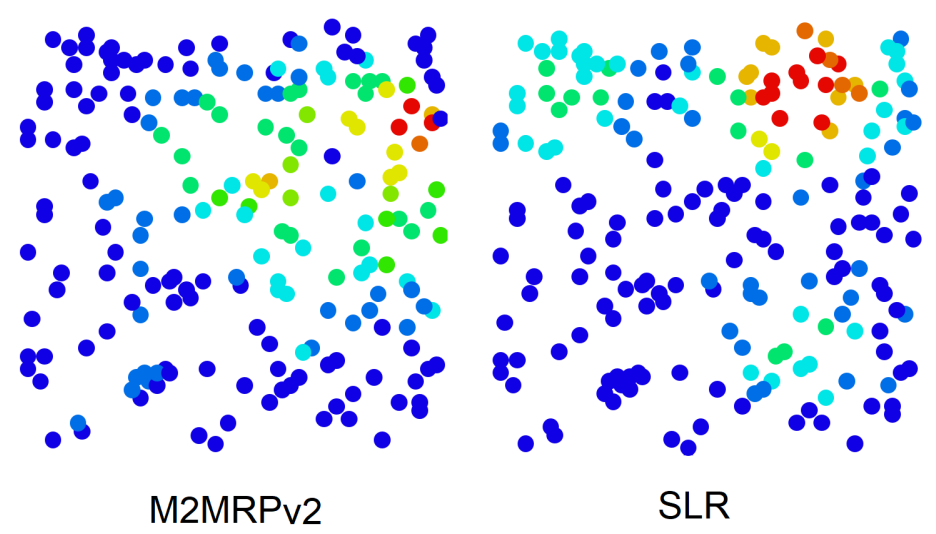

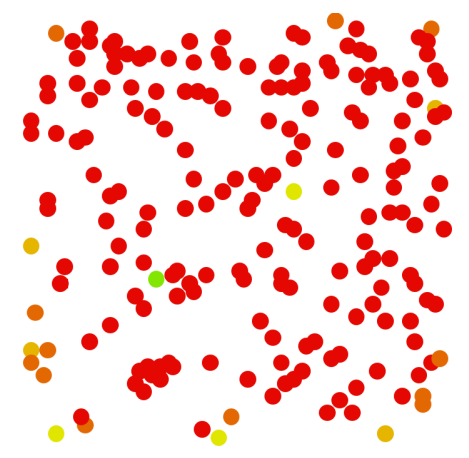

Broadcast
$<10 \%$

$<20 \%$

$<30 \%$

$<40 \%$

$<50 \%$

$<60 \%$

$<70 \%$

$<80 \%$

$<90 \%$

$>90 \%$

Figure 10: Batteries level after 4 routing cycles with three routing protocols: Broadcast, SLR, and M2MRPv2 protocols. Tests are made over a square topology nanonetwork with 200 nodes and an average traffic load of 0.2 packets per data cycle.

\subsection{Energy management}

Figure 10 shows the energy level of the network nanonodes starting with fully charged batteries after 4 routing cycles. We observe that the charge of batteries falls below $10 \%$ for all nodes with the broadcast method. Indeed, major nodes are used to relay every message. Whereas, SLR and M2MRPv2 protocols succeed in reducing the energy consumption which results in less discharged nanonodes with less than $70 \%$ of the full charge. However, we observe that M2MRPv2 protocol reduces the number of critical nanonodes presenting less than $10 \%$ of the charge, which underlines the quality of energy control in M2MRPv2 method.

Figure 11 shows the energy level cartography over the scenarios of figure 11 after 100 routing cycles. As shown in tests of the figure $8, M 2 M R P v 2$ succeeds to route 4 more times packets than SLR and Broadcast methods, which means more exchanged messages. Despite this, the energy level of nanonodes in M2MRPv2 is relatively identical to SLR approach and much better than the broadcast method. Indeed, M2MRPv2 adapts the data flow circulation to the state of charge of the nanonodes in each area, which allows overloaded nodes to recharge their batteries.

\section{Conclusions}

The routing in ultra-dense unstable nanonetwork is a novel problem. The classical approaches based on point-to-point relaying schemes are not relevant due to the nanonodes' uncertainty. on the other hand, the high density of the network and the nanonode constraints make some multirelay-to-multirelay approaches, such as OLSR, unusable.

In this paper, we presented a discussion about the expected memory and energy capabilities of the submillimeter nodes in order to determine the real limitations of the system. Then we compared two well-known multirelay-to-multirelay routing protocols with our new approach called M2MRPv2. The comparisons are made over scenarios representing different topological and traffic profiles. From these results, we deduce that M2MRPv2 protocol improves significantly the reliability of the system under energy-limited nanonodes. 

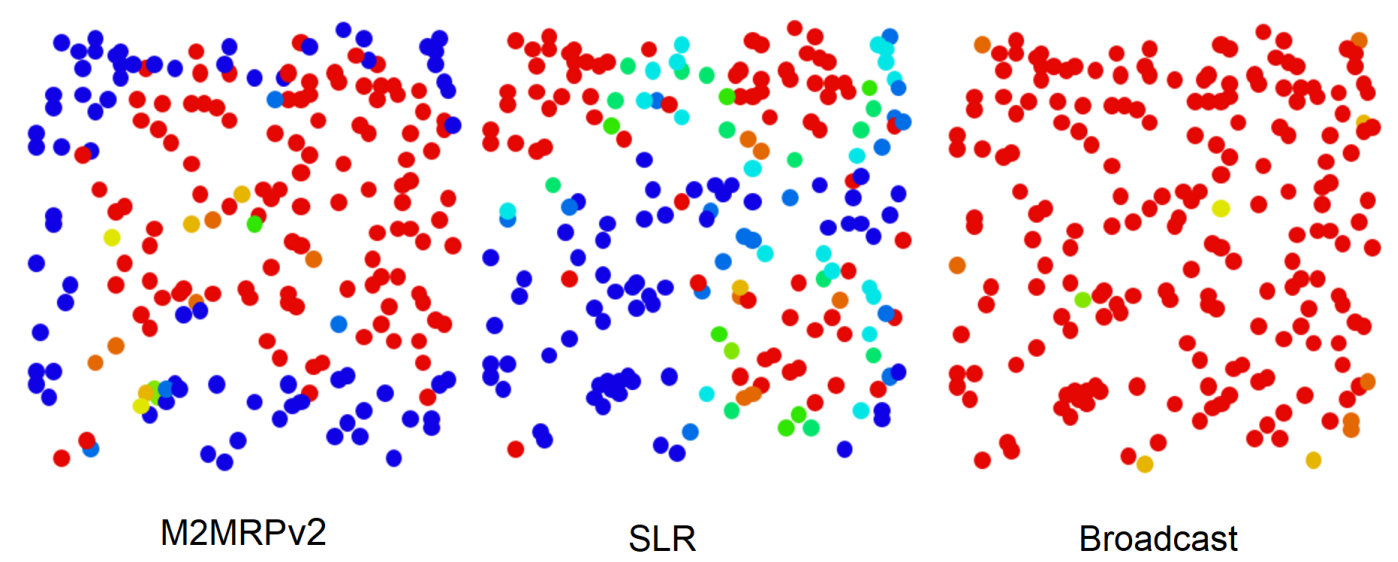

$<10 \%$

$<20 \%$

$<30 \%$

$<40 \%$

$<50 \%$

$<60 \%$

$<70 \%$

$<80 \%$

$<90 \%$

$>90 \%$

Figure 11: Batteries level after 100 routing cycles with three routing protocols: Broadcast, SLR, and M2MRPv2 protocols. Tests are made over a square topology nanonetwork with 200 nodes and an average traffic load of 0.2 packets per data cycle.

The paper addresses more particularly the Terahertz nanonetwork. But the M2MRPv2 protocol is clearly well adapted to any ultra-dense networks including dense sensor nanonetwork, IoT networks, and D2D 5G networks.

The optimization of the discharging/recharging cycles represents one of the important issues to address. Depending on the characteristics of the batteries, it could be preferable to completely discharge the battery before recharging it or, inversely, prevent the battery from being significantly discharged. Indeed, the way the battery is used impacts the service life duration of the batteries (number of discharging/recharging cycles).

\section{References}

[1] L. Aliouat, H. Mabed, J. Bourgeois, Flexible multipoint-to-multipoint routing protocol in ultra-dense nanonetworks, in: Proceedings of the 17th ACM International Symposium on Mobility Management and Wireless Access, 2019, pp. 81-87. doi:10.1145/3345770.3356746.

[2] C. Orsini, E. Gregori, L. Lenzini, D. Krioukov, Evolution of the internet k-dense structure, IEEE/ACM Transactions on Networking 22 (6) (2013) 1769-1780. doi:10.1109/TNET.2013.2282756.

[3] C. E. Perkins, E. M. Royer, Ad-hoc on-demand distance vector routing, in: Proceedings WMCSA'99. Second IEEE Workshop on Mobile Computing Systems and Applications, IEEE, 1999, pp. 90-100. doi:10.1109/MCSA.1999.749281.

[4] D. B. Johnson, D. A. Maltz, J. Broch, et al., Dsr: The dynamic source routing protocol for multi-hop wireless ad hoc networks, in: Ad hoc networking, Vol. 5, Addison Wesley, 2001, pp. 139-172.

[5] F. Al-Turjman, K. I. Kilic, Lagoon: a simple energy-aware routing protocol for wireless nano-sensor networks, IET Wireless Sensor Systems 9 (3) (2018) 110-118. doi:10.1049/iet-wss.2018.5079. 
[6] M. Pierobon, J. M. Jornet, N. Akkari, S. Almasri, I. F. Akyildiz, A routing framework for energy harvesting wireless nanosensor networks in the terahertz band, Wireless networks 20 (5) (2014) 11691183. doi:10.1007/s11276-013-0665-y.

[7] X.-W. Yao, Y. Yao, C.-F. Qi, W. Huang, et al., Eecr: Energy-efficient cooperative routing for embased nanonetworks, in: International Conference on Cooperative Design, Visualization and Engineering, Springer, 2019, pp. 30-38. doi:10.1007/978-3-030-30949-7_4.

[8] J. Cheng, Y. Gao, N. Zhang, H. Yang, An energy-efficient two-stage cooperative routing scheme in wireless multi-hop networks, Sensors 19 (5) (2019) 1002. doi:10.3390/s19051002.

[9] A. E. Abdallah, Smart partial flooding routing algorithms for 3d ad hoc networks, Procedia Computer Science 94 (2016) 264-271. doi:10.1016/j.procs.2016.08.040.

[10] T. Plesse, C. Adjih, P. Minet, A. Laouiti, A. Plakoo, M. Badel, P. Muhlethaler, P. Jacquet, J. Lecomte, Olsr performance measurement in a military mobile ad hoc network, Ad Hoc Networks 3 (5) (2005) 575-588. doi:10.1109/INMIC.2001.995315.

[11] A. Tsioliaridou, C. Liaskos, E. Dedu, S. Ioannidis, Packet routing in 3d nanonetworks: A lightweight, linear-path scheme, Nano communication networks 12 (2017) 63-71. doi:10.1016/j.nancom.2017.01.001.

[12] A. Tsioliaridou, C. Liaskos, S. Ioannidis, A. Pitsillides, Corona: A coordinate and routing system for nanonetworks, in: Proceedings of the second annual international conference on nanoscale computing and communication, Vol. 18, 2015, pp. 1-6. doi:10.1145/2800795.2800809.

[13] J. M. Jornet, I. F. Akyildiz, Femtosecond-long pulse-based modulation for terahertz band communication in nanonetworks, IEEE Transactions on Communications 62 (5) (2014) 1742-1754. doi:10.1109/TCOMM.2014.033014.130403.

[14] H. Ferjani, H. Touati, Efficient data dissemination in electromagnetic wireless nano-sensor networks, in: 2019 15th International Wireless Communications \& Mobile Computing Conference (IWCMC), IEEE, 2019, pp. 531-536. doi:10.1109/IWCMC.2019.8766554.

[15] F. Afsana, M. Asif-Ur-Rahman, M. R. Ahmed, M. Mahmud, M. S. Kaiser, An energy conserving routing scheme for wireless body sensor nanonetwork communication, IEEE Access 6 (2018) 9186-9200. doi:10.1109/ACCESS.2018.2789437.

[16] L. Aliouat, H. Mabed, J. Bourgeois, 2.5 layer protocol for traffic regulation in ultra-dense nanonetwork, in: International Conference on Ad-Hoc Networks and Wireless, Springer, 2019, pp. 330-340. doi:10.1007/978-3-030-31831-4_23. 
[17] E. V. Aleksandrova, V. A. Bashkin, Hydrodynamic adaptive routing algorithm for unstable sensor networks with a tsunami model of acute events, in: SENSORNETS, 2018, pp. 141-146. doi:10.5220/0006655901410146.

[18] N. Gupta, R. Gupta, Routing protocols in mobile ad-hoc networks: an overview, in: INTERACT-2010, IEEE, 2010, pp. 173-177. doi:10.1109/INTERACT.2010.5706220.

[19] F. Goudarzi, H. Asgari, H. S. Al-Raweshidy, Traffic-aware vanet routing for city environmentsa protocol based on ant colony optimization, IEEE Systems Journal 13 (1) (2018) 571-581. doi:10.1109/JSYST.2018.2806996.

[20] M. R. Senouci, A. Mellouk, H. Senouci, A. Aissani, Performance evaluation of network lifetime spatialtemporal distribution for wsn routing protocols, Journal of Network and Computer Applications 35 (4) (2012) 1317-1328. doi:10.1016/j.jnca.2012.01.016.

[21] S. R. Malwe, N. Taneja, G. Biswas, Enhancement of dsr and aodv protocols using link availability prediction, Wireless Personal Communications 97 (3) (2017) 4451-4466. doi:10.1007/s11277-017-4733-y.

[22] U. R. Bhatt, N. Nema, R. Upadhyay, Enhanced dsr: An efficient routing protocol for manet, in: 2014 International Conference on Issues and Challenges in Intelligent Computing Techniques (ICICT), IEEE, 2014, pp. 215-219. doi:10.1109/ICICICT.2014.6781282.

[23] N. Abuali, S. Aleyadeh, F. Djebbar, A. Alomainy, M. M. A. Almaazmi, S. Al Ghaithi, Performance evaluation of routing protocols in electromagnetic nanonetworks, IEEE Access 6 (2018) 35908-35914. doi:10.1109/ACCESS.2018.2845305.

[24] M. Appiah, R. Cudjoe, A comparative study of reactive and proactive routing protocols on a mobility model in mobile ad hoc network (manet), in: 2018 International Conference on Smart Computing and Electronic Enterprise (ICSCEE), IEEE, 2018, pp. 1-7. doi:10.1109/ICSCEE.2018.8538398.

[25] G. D. Kondylis, S. V. Krishnamurthy, S. K. Dao, G. J. Pottie, Multicasting sustained cbr and vbr traffic in wireless ad-hoc networks, in: 2000 IEEE International Conference on Communications. ICC 2000. Global Convergence Through Communications. Conference Record, Vol. 1, IEEE, 2000, pp. 543549. doi:10.1109/ICC.2000.853377.

[26] C. Feuillet-Palma, Y. Todorov, A. Vasanelli, C. Sirtori, Strong near field enhancement in thz nanoantenna arrays, Scientific reports 3 (1) (2013) 1-8. doi:10.1038/srep01361.

[27] J. W. Judy, Microelectromechanical systems (MEMS): fabrication, design and applications, Smart materials and Structures 10 (6) (2001) 1115. doi:10.1088/0964-1726/10/6/301. 
[28] J. Bourgeois, B. Piranda, A. Naz, N. Boillot, H. Mabed, D. Dhoutaut, T. Tucci, H. Lakhlef, Programmable matter as a cyber-physical conjugation, in: 2016 IEEE International Conference on Systems, Man, and Cybernetics (SMC), IEEE, 2016, pp. 2942-2947. doi:10.1109/SMC.2016.7844687.

[29] N. Boillot, D. Dhoutaut, J. Bourgeois, Using nano-wireless communications in micro-robots applications, in: Proceedings of ACM the first annual international conference on nanoscale computing and communication, ACM, 2014, pp. 1-9. doi:10.1145/2619955.2619967.

[30] S. Canovas-Carrasco, R. M. Sandoval, A.-J. Garcia-Sanchez, J. Garcia-Haro, Optimal transmission policy derivation for iont flow-guided nano-sensor networks, IEEE Internet of Things Journal 6 (2)(2019) 2288-2298. doi:10.1109/JIOT.2019.2906015.

[31] A. Rahim, P. Malone, Intrusion detection system for wireless nano sensor networks, in: 8th International Conference for Internet Technology and Secured Transactions (ICITST-2013), IEEE, 2013, pp. 327-330. doi:10.1109/ICITST.2013.6750215.

[32] V. Upadhayay, S. Agarwal, Application of wireless nano sensor networks for wild lives, International Journal of Distributed and Parallel systems 3 (4) (2012) 173. doi:10.5121/ijdps.2012.3418.

[33] M. Ding, D. López-Pérez, On the performance of practical ultra-dense networks: The major and minor factors, in: 2017 15th International Symposium on Modeling and Optimization in Mobile, Ad Hoc, and Wireless Networks (WiOpt), IEEE, 2017, pp. 1-8. doi:10.23919/WIOPT.2017.7959926.

[34] H. Yu, B. Ng, W. Seah, Multi-gateway polling for nanonetworks under dynamic iot backhaul bandwidth, in: 2018 IEEE International Conference on Sensing, Communication and Networking (SECON Workshops), IEEE, 2018, pp. 1-4. doi:10.1109/SECONW.2018.8396353.

[35] J. C. Pujol, J. M. Jornet, J. S. Pareta, Phlame: A physical layer aware mac protocol for electromagnetic nanonetworks, in: 2011 IEEE Conference on Computer Communications Workshops (INFOCOM WKSHPS), IEEE, 2011, pp. 4314-36. doi:10.1109/INFCOMW.2011.5928852.

[36] O. J. Adinya, L. Daoliang, Transceiver energy consumption models for the design of low power wireless sensor networks, in: 2012 IEEE Student Conference on Research and Development (SCOReD), IEEE, 2012, pp. 193-197. doi:10.1109/SCOReD.2012.6518637.

[37] J. N. Dehankar, P. Patil, G. Agarwal, Survey on energy consumption in wireless sensor network, International Journal of Engineering Research Technology (IJERT) 2 (1) (2014) 1-4, https://www.ijert.org/ research/survey-on-energy-consumption-in-wireless-sensor-network-IJERTV2IS1071.pdf.

[38] R. E. Fontana Jr, G. M. Decad, Moore's law realities for recording systems and memory storage components: Hdd, tape, nand, and optical, AIP Advances 8 (5) (2018) 056506. doi:10.1063/1.5007621. 
[39] H. Acar, G. I. Alptekin, J.-P. Gelas, P. Ghodous, Beyond cpu: Considering memory power consumption of software, in: 2016 5th International Conference on Smart Cities and Green ICT Systems (SMARTGREENS), IEEE, 2016, pp. 1-8. doi:10.5220/0005764904170424.

[40] P. Visconti, P. Primiceri, R. Ferri, M. Pucciarelli, E. Venere, An overview on state-of-art energy harvesting techniques and choice criteria: a wsn node for goods transport and storage powered by a smart solar-based eh system, International Journal of Renewable Energy Research (IJRER) 7 (3) (2017) 12811295, https://www.ijrer.org/ijrer/index.php/ijrer/article/view/6052/pdf.

[41] S. Boisseau, G. Despesse, B. A. Seddik, Electrostatic conversion for vibration energy harvesting, SmallScale Energy Harvesting (2012) 1-39. doi:10.5772/51360.

[42] S. P. Beeby, T. O’Donnell, Electromagnetic energy harvesting, in: Energy Harvesting Technologies, Springer, 2009, pp. 129-161. doi:10.1007/978-0-387-76464-1_5.

[43] G. Varshney, Reconfigurable graphene antenna for thz applications: a mode conversion approach, Nanotechnology 31 (13) (2020) 135208. doi:10.1088/1361-6528/ab60cc.

[44] X. Fu, F. Yang, C. Liu, X. Wu, T. J. Cui, Terahertz beam steering technologies: From phased arrays to Field-programmable metasurfaces, Advanced Optical Materials 8 (3) (2020) 1900628. doi:10.1002/adom.201900628. 